\title{
Research on Recognition and Application of EEG Signal Based on SSVEP-BCI
}

\author{
Di Yin ${ }^{1}$, Fengzhi Dai ${ }^{1,3 *}$, Mengqi Yin', Yasheng Yuan', Yuxuan Zhu' \\ ${ }^{1}$ Tianjin University of Science and Technology, China; \\ ${ }^{2}$ Hebei University of Chinese Medicine, China; \\ ${ }^{3}$ Tianjin Tianke Intelligent and Manufacture Technology CO., LTD, China, \\ E-mail:*daifz@tust.edu.cn \\ www.tust.edu.cn
}

\begin{abstract}
In recent years, brain-computer interface (BCI) systems based on steady-state visual evoked potentials (SSVEP) have attracted attention due to their high information transfer rate (ITR) and more and more targets. The current mainstream algorithms for SSVEP recognition have greatly improved the accuracy and target detection time. This paper designs a robotic arm application system based on the eCCA-Y method for multi-target recognition. The phase characteristics of CCA's sine and cosine signals are added to the EEG signal. Compared with mainstream algorithms, research shows that this method can improve the SSVEP-based BCI performance. And choose a six-degree-of-freedom manipulator as the actuator of the brain-computer interface, and use a phase-encoded stimulation paradigm for multi-target recognition to conduct experiments on the application of the proposed method.
\end{abstract}

Keywords: Brain-computer interface, Feature extraction, Multi-target recognition, EEG signal, Steady state visual evoked potential

\section{Introduction}

Patients suffering from diseases such as muscular dystrophy and spinal injury have physical disabilities but normal brain functions, but they cannot interact with the outside world like normal people. Therefore, an output channel can be created directly by the brain to the external environment, without communicating through peripheral nerves and muscle tissues ${ }^{1,2}$. Brain-computer interface technology is booming and can meet this demand. The brain signals issue commands to directly control external devices, so that patients can restore their body functions and live like normal people.

In the research of brain-computer interface, the feature extraction algorithm of EEG signal is to find a suitable spatial filter, and get the weight vector through projection and linear combination. Zhang et.al proposed the multi-channel $\mathrm{CCA}^{3}$. Wong et al. proposed eCCA ${ }^{4}$, Masaki et al. introduced the task-related component analysis (TRCA) method to SSVEP identification ${ }^{5}$.

We found that no phase information is seen in the sine and cosine reference signal, that is, there is no trace of phase information in the constructed fitting signal, so an algorithm based on eCCA (eCCA-Y) ${ }^{6}$, in the SSVEP stimulation paradigm is proposed. The phase is added to the reference signal, and the phase of the reference signal is obtained by optimizing the parameters. Compared with the existing two mainstream algorithms, the recognition rate and ITR have a certain improvement.

In terms of application research, the EEG signal is used to control the robotic arm equipment to verify the effectiveness of the algorithm, complete the communication establishment of the two subsystems, and the EEG signal instructions are converted into motion 
control instructions for controlling the end effector of the robotic arm.

\section{Application of EEG Signal Recognition Based on SSVEP-BCI System}

According to the experimental intent of the stimulus paradigm and the current position of the robotic arm, the participants feedback and select the control instructions of the experimental paradigm. At the same time, the EEG acquisition equipment performs a series of processing (preprocessing, feature extraction and feature recognition). Map the recognized instructions to the actual robotic arm commands, and the robotic arm executes actions according to the received instructions.

As shown in Fig.1, it mainly consists of four parts: EEG signal acquisition module, signal decoding module, human-computer interaction module and command output module.

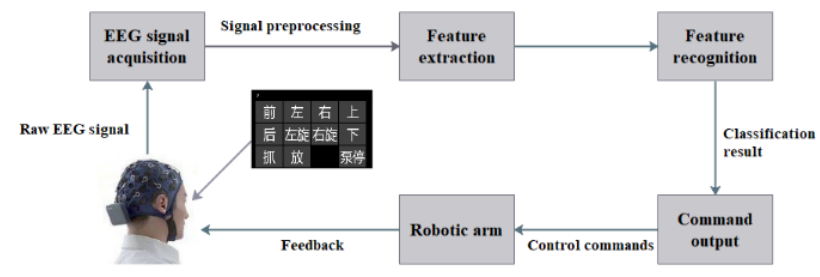

Fig.1 Application flow chart based on SSVEP-BCI system

\section{Experiment}

\subsection{Experimental paradigm}

The EEG acquisition system based on SSVEP-BCI compiles some inherent actions of the robotic arm through MATLAB's PSYCHTOOLBOX (PTB) toolbox to form a specific stimulation paradigm. The stimulus paradigm formed at the end of this experiment is shown in Fig.2.

\begin{tabular}{|r|r|r|r|}
\hline 9 & 9.25 & 9.5 & 9.75 \\
0 & $0.5 \pi$ & $\pi$ & $1.5 \pi$ \\
\hline 10.25 & 10.5 & 10.75 & 11 \\
0 & $0.5 \pi$ & $\pi$ & $1.5 \pi$ \\
\hline 11.25 & 11.50 & & 11.75 \\
0 & $0.5 \pi$ & & $1.5 \pi$ \\
\hline
\end{tabular}

Fig.2 Manipulator stimulation paradigm based on SSVEP-BCI control

\subsection{The experimental data}

The data set collected SSVEP-BCI records of 5 healthy subjects (4 males and 1 female), the average age of the subjects was 24 years old. Before starting, the two subjects who have been tested before will be trained with data. For each subject, a total of 5 blocks were performed, each with 11 targets. The stimulation paradigm has been shown in Fig.2.

The multi-target recognition sinusoidal coding method was used to accurately generate 11 stimulation frequencies on the LCD display. Just as shown in Fig.2, the location of the eleven targets on the screen stimulation frequency corresponds to $9 \mathrm{~Hz}, 9.25 \mathrm{~Hz}, 9.5 \mathrm{~Hz}$, $9.75 \mathrm{~Hz}, 10.25 \mathrm{~Hz}, 10.5 \mathrm{~Hz}, 10.75 \mathrm{~Hz}, 11 \mathrm{~Hz}, 11.25 \mathrm{~Hz}$, $11.5 \mathrm{~Hz}$ and $11.75 \mathrm{~Hz}$.

Traverse 11 targets in turn, each target stimulus is $5 \mathrm{~s}$ (including stimulus prompt $0.5 \mathrm{~s}$, stimulus flashing $4.5 \mathrm{~s}$ ). Participants try to avoid blinking each time the stimulus target blinks. Each time the stimulus target blinks, the subjects try their best to avoid blinking, so after the end of each group of experiments rest for 2-3 minutes in between. The experimental arrangement of the test data is shown in Fig.3.

\begin{tabular}{|c|c|c|c|c|c|c|c|}
\hline $0.5 \mathrm{~s}$ & $4.5 \mathrm{~s}$ & $0.5 \mathrm{~s}$ & $4.5 \mathrm{~s}$ & $0.5 \mathrm{~s}$ & $\cdots$ & $0.5 \mathrm{~s}$ & $4.5 \mathrm{~s}$ \\
\hline & $9 \mathrm{~Hz}$ & & $9.25 \mathrm{~Hz}$ & & $\ldots$ & & $11.75 \mathrm{~Hz}$ \\
\hline & & & rial2 & & & & trial11 \\
\hline \multicolumn{8}{|c|}{$55 \mathrm{~s}$} \\
\hline
\end{tabular}

Fig.3 Experimental arrangement of test data

\subsection{Data preprocessing}

Preprocess the collected EEG data in the EEGLAB toolbox of MATLAB software, extract 8 channels (Pz, $\mathrm{PO} 3, \mathrm{POz}, \mathrm{PO} 4, \mathrm{PO}, \mathrm{O} 1, \mathrm{Oz}$, and $\mathrm{O} 2$ ), and get the brain electrical three-dimensional data (channel*points*trial).

In order to make the collected EEG data more convincing in the subsequent feature extraction and analysis process, the number of the experimental groups (block) is merged into the preprocessed three-dimensional EEG data and thus obtain the four-dimensional data (channel*points*trial*block). Down sampling is performed from $1000 \mathrm{~Hz}$ to $250 \mathrm{~Hz}$, and each trial contains 3000 sampling points, which forms $8 * 750 * 11 * 5$ EEG data.

\section{Feature extraction}


When the subjects are training data, they can obtain three kinds of multi-channel signals:

1. Test data $X(t) \in R^{N_{C} \times N_{S} \times N_{t}}$

2. The average signal of the subject's training data $\hat{X}_{k}{ }^{N_{c} \times N_{s}}$

3. Constructed sine and cosine reference signal $Y_{f k}$

Among them, $N_{C}, N_{S}, N_{t}$ represent channel, points, and test set trial respectively. Any two signals can be used to calculate a spatial filter based on CCA.

Chen et al. $^{7}$ combined the CCA system with the Pearson correlation coefficient of the test training data of the subjects and proposed a method to extend CCA. Only three spatial filter forms are taken, namely:

1. Test data and average training data $W_{X}\left(X \hat{X}_{k}\right), W_{\hat{X}_{k}}\left(X \hat{X}_{k}\right)$

2. Test data and reference signal $W_{X}\left(X Y_{f k}\right)$

3. Average training data and reference signal $W_{\hat{X}_{k}}\left(\hat{X}_{k} Y_{f k}\right)$

According to the above three spatial filters, four correlation coefficient combinations as in formula (1) are selected:

$$
r_{k}=\left[\begin{array}{c}
r_{k}(1) \\
r_{k}(2) \\
r_{k}(3) \\
r_{k}(4)
\end{array}\right]=\left(\begin{array}{l}
\rho\left(X^{T} W_{X}\left(X Y_{f k}\right), Y_{f k}^{T} W_{Y_{f k}}\left(X Y_{f k}\right)\right) \\
\rho\left(X^{T} W_{X}\left(X \hat{X}_{k}\right), \hat{X}_{k}^{T} W_{X}\left(X \hat{X}_{k}\right)\right) \\
\rho\left(X^{T} W_{X}\left(X Y_{f k}\right), \hat{X}_{k}^{T} W_{X}\left(X Y_{f k}\right)\right) \\
\rho\left(X^{T} W_{\hat{X}_{k}}\left(\hat{X}_{k} Y_{f k}\right), \hat{X}_{k}^{T} W_{\hat{X}_{k}}\left(\hat{X}_{k} Y_{f k}\right)\right)
\end{array}\right)
$$

The correlation coefficient is fused to obtain the correlation coefficient at the k-th stimulus frequency, and then the classification can be completed by selecting the largest correlation coefficient. The principle analysis diagram is shown in Fig. 4.

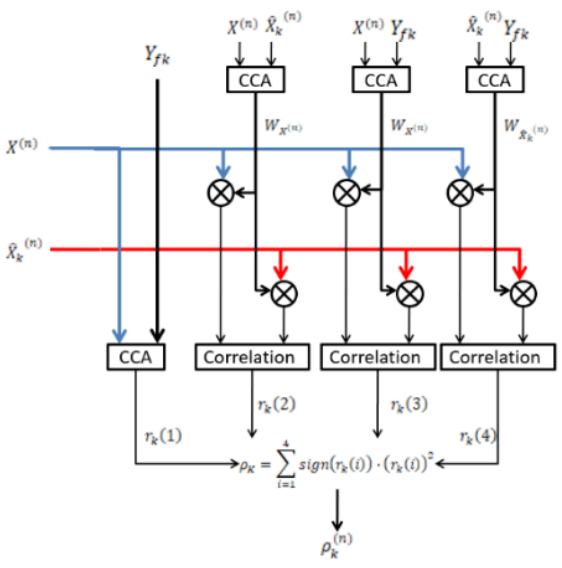

The reference signal is:

$$
Y_{f k, \theta_{k}}=\left[\begin{array}{c}
\sin \left(2 \pi f_{k} t+\theta_{k}\right) \\
\cos \left(2 \pi f_{k} t+\theta_{k}\right) \\
\vdots \\
\sin \left(2 \pi N_{h} f_{k} t+N_{h} \theta_{k}\right) \\
\cos \left(2 \pi N_{h} f_{k} t+N_{h} \theta_{k}\right)
\end{array}\right]^{T}
$$

According to the experimental intent, the stimulus paradigm designed by the researcher determines the size of $\theta_{k}$, as expressed by formula (3):

$$
\theta_{k}=\theta_{0}+\Delta \theta \times\left[\left(k_{y}-1\right) \times 5+\left(k_{x}-1\right)\right]
$$

Among them, $k_{x}$ and $k_{y}$ represent the row and column indices of the visual stimulation matrix, respectively. $\theta_{0}$ represents the initial phase, and $\Delta \theta$ represents the phase interval.

\section{Research on Application of Manipulator Based on SSVEP-BCI}

The robotic arm application based on SSVEP-BCI is composed of two systems, namely the BCI EEG acquisition system and the robotic system. The BCI subsystem and the robot subsystem communicate through the UDP protocol. The BCI subsystem is composed of a wireless EEG acquisition system, LCD display and online processing of EEG data.

The EEG acquisition equipment is responsible for converting the recorded EEG signals into digital signals through $\mathrm{AD}$, and through online real-time processing (feature extraction and conversion algorithms) to generate control instructions that can be recognized by the robotic arm. The reason for joining the application of robotic arm in this article is to study whether the robotic arm can provide convenience for the disabled and solve the simple grasping movements necessary in life.

The steps of the application design in this article are: (1) collect the subject's EEG data and record it as the subject's EEG intent, (2) perform data processing and analysis on the saved data in the MATLAB software, (3) and finally convert it into the control command of the robotic arm and send it to the controller.

In this experiment, the EEG data of 5 students in school was collected, feature extraction and recognition and classification were performed, and finally it was transformed into instructions that can control the robotic arm and sent to the robotic arm controller.

Fig.4 eCCA-Y schematic diagram

(C) The 2021 International Conference on Artificial Life and Robotics (ICAROB2021), January 21 to 24, 2021 
This experiment analyzes the data of 5 subjects. The number of harmonics is 2 and the data length is 750 sampling points to compare the classification effects of the two algorithms eCCA and eCCA-Y, as shown in Fig.5.
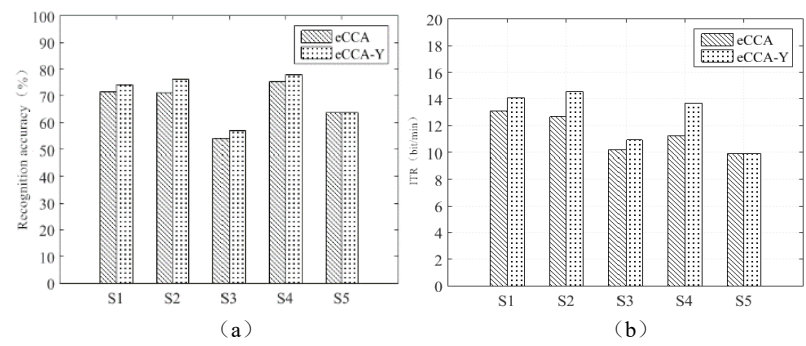

Fig.5 eCCA-Y schematic diagram

It can be seen from Fig.5(a) that the optimized eCCA-Y algorithm in this paper has improved recognition rate. Among them, S5 has the same accuracy rate for the two algorithms, and the recognition rate of S3 is relative to the other four. The test is low, S2 subjects have the largest difference in recognition rates between the two algorithms. In terms of ITR, as shown in Fig.5(b), the eCCA-Y algorithm is also improved, and S5 also shows the same effect as the recognition accuracy. The two algorithms are the same in terms of ITR. In addition, S4 subjects have the largest difference in ITR for the two algorithms.

\section{Conclusion}

Based on the SSVEP-BCI system to carry out application research experiments on the robotic arm, a multi-target recognition stimulus paradigm for the robotic arm is designed. The UDP protocol is used to realize the communication between the robotic arm and the brain-computer interface system. It is added at different frequencies. Different phases simplified the control method, refined the control of the robotic arm movement, and weakened its complexity.

Two feature recognition algorithms, eCCA and eCCA-Y, are used to process data, verifying the robotic arm and the brain-computer interface. The application realizes the interactive control of the robotic arm, and adds a stroke to the feature processing algorithm, which provides experience for the development of the BCI system.

\section{Acknowledgements}

The research is partly supported by the Project of Tianjin Enterprise Science and Technology Commissioner to Tianjin Tianke Intelligent and Manufacture Technology Co., Ltd (19JCTPJC53700). It is also supported by the Industry-University Cooperation and Education Project (201802286009) from Ministry of Education, China.

\section{References}

1. Chang H C, Lee P L, Lo M T, et al. Independence of amplitude-frequency and phase calibrations in an SSVEP-based BCI using stepping delay flickering sequences. IEEE Transactions on Neural Systems and Rehabilitation Engineering, 2011, 20(3) : pp.305-312.

2. Mak J N, Wolpaw J R. Clinical applications of brain-computer interfaces: current state and future prospects. IEEE reviews in biomedical engineering, 2009, 2: pp.187-199.

3. Zhang Y.et al. Multiway canonical correlation analysis for frequency components recognition in SSVEP-based BCIs. International conference on neural information processing. Springer, Berlin, Heidelberg, 2011: pp.287-295.

4. Wong CM, Wan F, Wang B, et. al. Learning across multi-stimulus enhances target recognition methods in SSVEP-based BCIs. J. Neural Engineering, 2020, 17: 016026.

5. Nakanishi M, Wang YJ, Chen XG, Wang YT, Gao XR, Jung TP. Enhancing detection of ssveps for a high-speed brain speller using task-related component analysis. IEEE Transactions on Biomedical Engineering, 2018, 65: pp. 104-112.

6. Yin D, Dai F, Song Y, et al. On Improvement of Target Recognition eCCA Method Based on SSVEP. Chinese Intelligent Systems Conference. Springer, Singapore, 2020: pp. 742-749.

7. Chen X, Wang Y, Nakanishi M, et al. High-speed spelling with a noninvasive brain-computer interface. Proceedings of the national academy of sciences, 2015, 112(44): pp. E6058-E6067. 